\title{
Line formation in solar granulation
}

\section{Missing UV-opacity and the photospheric Be abundance}

\begin{abstract}
M. Asplund ${ }^{\star}$
Research School of Astronomy and Astrophysics, Mount Stromlo Observatory, Cotter Road, Weston, ACT 2611, Australia

Received 17 September 2003 / Accepted 3 November 2003

Abstract. The possibility of unaccounted for opacity sources in the UV for late-type stars has often been invoked to explain discrepancies between predicted and observed flux distributions and spectral line strengths. Such missing UV-opacity could among other things have a significant impact on abundance determination for elements whose only relevant spectral features are accessible in this wavelength region, such as Be. Here, the study by Balachandran \& Bell (1998) is re-visited in the light of a realistic 3D hydrodynamical solar model atmosphere and the recently significantly downward revised solar $\mathrm{O}$ abundance obtained with the same model atmosphere. The amount of missing UV-opacity, if any, is quantified by enforcing that the $\mathrm{OH}$ A-X electronic lines around $313 \mathrm{~nm}$ produce the same $\mathrm{O}$ abundance as the other available diagnostics: $\mathrm{OH}$ vibration-rotation and pure rotation lines in the IR, the forbidden [O I] 630.0 and $636.3 \mathrm{~nm}$ lines and high-excitation, permitted O I lines. This additional opacity is then applied for the synthesis of the Be II line at $313.0 \mathrm{~nm}$ to derive a solar photospheric Be abundance in excellent agreement with the meteoritic value, thus re-enforcing the conclusions of Balachandran \& Bell. The about 50\% extra opacity over accounted for opacity sources can be well explained by recent calculations by the Iron Project for photo-ionization of Fe I.
\end{abstract}

Key words. convection - line: formation - Sun: abundances - Sun: granulation - Sun: photosphere

\section{Introduction}

Among other things, the light elements lithium, beryllium and boron offer an opportunity to extract information about stellar interiors and evolution. Due to their fragile nature, these elements are destroyed by nuclear processing when brought to sufficiently high temperatures by stellar convection or other mixing events. Since the destruction of $\mathrm{Li}, \mathrm{Be}$ and $\mathrm{B}$ occurs at somewhat different temperature regimes $\left(\sim 2.6 \times 10^{6} \mathrm{~K}\right.$ for ${ }^{7} \mathrm{Li}$, $\sim 3.5 \times 10^{6} \mathrm{~K}$ for Be and $\sim 5 \times 10^{6} \mathrm{~K}$ for B), the relative amount of depletion of these elements can function as sensitive probes how deep the stellar mixing has proceeded. Indeed there is extensive information available on light element depletion in stars of different spectral types (e.g. Deliyannis et al. 2000), confirming the presence of additional mixing over that predicted by the standard mixing length theory for convection during the pre-main sequence and main sequence evolution. Among the invoked explanations for the observed depletion patterns, slow rotationally-induced mixing has perhaps received the most attention recently given the reasonable agreement with observations for its predictions over a range of stellar environments (Deliyannis et al. 2000).

Neither has our Sun been immune to light element depletion. The observed photospheric $\mathrm{Li}$ abundance is about a factor of 140 lower than measured in CI-chondrite meteorites

\footnotetext{
* e-mail: martin@mso.anu.edu.au
}

(Müller et al. 1975; Kiselman 1997; Asplund et al. 2003a; Lodders 2003), a difference which is far beyond the uncertainties involved in the photospheric and meteoritic abundance determinations. Until recently, it was believed that also Be and B were depleted in the solar photosphere by factors of about 1.8 (Chiemlewski et al. 1975) and 1.9 (Kohl et al. 1977; Kiselman \& Carlsson 1996), respectively, relative to the meteoritic evidence. This standard picture was challenged by Balachandran \& Bell (1998; see also Bell et al. 2001), who attempted to calibrate the since-long suspected missing UV-opacity (e.g. Vernazza et al. 1976; Gustafsson \& Bell 1979) by enforcing that the same oxygen abundance should be determined from the $\mathrm{OH}$ A-X electronic lines around the $313 \mathrm{~nm}$ region, where the crucial Be II lines are located, as the more reliable IR vibrationrotation counterparts of $\mathrm{OH}\left(\log \epsilon_{\mathrm{O}}=8.91^{1}\right.$ in the case of the 1D Holweger-Müller solar model atmosphere). Their investigation indicated a missing opacity for these wavelengths of about $60 \%$ over known opacity sources, which they attributed to photo-ionization of Fe I. Indeed, subsequent analysis of detailed atomic calculations performed within the framework of the Iron Project (Hummer et al. 1993) indicated that this hypothesis was quite plausible (Bell et al. 2001). When accounting for this missing continuous opacity in the synthesis of the

${ }^{1}$ On the customary logarithmic abundance scale defined to have a hydrogen abundance of $\log \epsilon_{\mathrm{H}}=12.00$. 
Be II doublet at $313 \mathrm{~nm}$, Balachandran \& Bell (1998) found that the resulting photospheric $\mathrm{Be}$ abundance was indistinguishable from the commonly adopted meteoritic Be abundance of $\log \epsilon_{\mathrm{Be}}=1.41$ (Lodders 2003). Furthermore, both the photospheric and meteoritic $\mathrm{B}$ abundances have recently been revised, bringing them into apparent agreement within their (substantial) uncertainties (Cunha \& Smith 1999; Zhai \& Shaw 1994). Current thinking thus implies that while Li in the solar convection zone has been significantly destroyed, neither Be nor B have been depleted relative to their proto-solar values. As indicated above, such a depletion pattern requires additional non-standard mixing, although the precise nature of this mixing is still eluding astronomers.

It is important to realise that the influential analysis of Balachandran \& Bell (1998) hinges on at least six crucial assumptions:

1. the standard 1D hydrostatic solar model atmospheres employed in the analysis provide a realistic description of the line formation;

2. the adopted transition probabilities for the $\mathrm{OH}$ lines are correct;

3. the adopted microturbulence is appropriate for these partly saturated $\mathrm{OH}$ A-X lines;

4. the continuum placement in the very crowded UV region of the $\mathrm{OH}$ lines is correct;

5. local thermodynamic equilibrium (LTE) for the $\mathrm{OH}$ line formation and equilibrium chemistry for the $\mathrm{OH}$ molecule formation are justified, and

6. LTE is good approximation for the Be II line formation.

Addressing some of these lingering potential sources of errors is now possible. Recently, the new generation of 3D hydrodynamical model atmospheres (Stein \& Nordlund 1998; Asplund et al. 1999, 2000a; Asplund \& García Pérez 2001) has been applied to studies of spectral line formation in the solar atmosphere for abundance determinations (Asplund 2000; Asplund et al. 2000b, 2003a,b,c,d; Allende Prieto et al. 2001, 2002b; Shchukina \& Trujillo Bueno 2001). A particularly noteworthy finding from these studies is the substantial downward revision of the solar photospheric $\mathrm{O}$ abundance to $\log \epsilon_{\mathrm{O}}=8.66 \pm 0.05$ (Asplund et al. 2004). This new abundance has been determined from a multitude of different spectral lines and for the first time all $\mathrm{O}$ diagnostics yield consistent results: the forbidden [OI] line at $630 \mathrm{~nm}$, high-excitation, permitted $\mathrm{OI}, \mathrm{OH}$ vibration-rotation lines and $\mathrm{OH}$ pure rotation lines. In view of the significantly lower $\mathrm{O}$ abundance commonly accepted today (Lodders 2003) compared with the value believed to be correct at the time of the study by Balachandran \& Bell (1998), it is clearly of importance to repeat their analysis using the new, highly realistic, 3D hydrodynamical solar model atmosphere. Such calculations are presented here, basically confirming the findings of Balachandran \& Bell (1998) of significant ( 50\%) missing UV-opacity and a photospheric Be abundance in agreement with the meteoritic abundance. Of the above-mentioned six uncertainties in the original Balachandran \& Bell (1998) study, apparently now only the penultimate one remains which could possibly modify these conclusions.

\section{3D spectral line formation calculations}

The same 3D hydrodynamical solar model atmosphere which has previously been confronted successfully with a range of observational diagnostics (e.g. Asplund et al. 2000a) and used for abundance analysis purposes (Asplund 2000; Asplund et al. 2000b, 2003a,b,c,d; Allende Prieto et al. 2001, 2002b; Shchukina \& Trujillo Bueno 2001) is employed here. For numerical details of the simulation, the reader is referred to Stein \& Nordlund (1998), Asplund et al. (2000a) and Asplund \& García Pérez (2001).

The 3D spectral line formation calculations are performed under the assumption of LTE for the ionization and excitation balances and for the source function $\left(S_{v}=B_{v}\right)$. Instantaneous chemical equilibrium is furthermore assumed for the $\mathrm{OH}$ molecule formation. The radiative transfer is solved for 17 different inclined directions $\left(N_{\mu}=N_{\varphi}=4\right.$ plus the vertical) using realistic background continuous opacities (Gustafsson et al. 1975; Asplund et al. 1997) and an equation-of-state which accounts for excitation, ionization and molecule formation of the most important elements (Mihalas et al. 1988). The photo-ionization cross-sections of Fe I as calculated by the Iron Project (Hummer et al. 1993) have not been included. It is likely that the missing UV-opacity quantified below is indeed due to Fe I bound-free opacity (Bell et al. 2001). The inclined rays are disk-integrated adopting a solar rotational velocity of $1.8 \mathrm{~km} \mathrm{~s}^{-1}$ to produce flux profiles; the exact choice of rotational broadening is however inconsequential for this study. The instantaneous flux profiles are subsequently averaged over a simulation time-sequence corresponding to about $50 \mathrm{~min}$ of solar time, which is sufficiently long to yield statistically significant results.

A major advantage with the new generation of 3D hydrodynamical model atmospheres is that the traditional free parameters of stellar spectroscopy (mixing length parameters to describe convection and the micro- and macroturbulence for line broadening) no longer are needed due to the self-consistently calculated convective velocities and the corresponding Doppler shifts (Asplund et al. 2000a,b; Asplund 2000; Allende Prieto et al. 2002a). Thus, no microturbulence $\xi_{\text {turb }}$ enters the 3D calculations presented herein, although the corresponding 1D calculations have been performed with both $\xi_{\text {turb }}=1.0 \mathrm{~km} \mathrm{~s}^{-1}$ (the standard case as well as the value adopted by Balachandran \& Bell 1998) and $\xi_{\text {turb }}=1.2 \mathrm{~km} \mathrm{~s}^{-1}$, to assess the uncertainties attached to the adopted microturbulence in the original analysis of Balachandran \& Bell (1998).

The molecular data for the $\mathrm{OH}$ and $\mathrm{Be}$ lines are wellknown. Here a small selection of four OH A-X lines are chosen $(\mathrm{OH} 312.80,312.82,313.91$ and $316.71 \mathrm{~nm})$ covering the relevant range of excitation potential. As will be evident later on, all lines suggest very similar multiplication factors to the opacity at these wavelengths, and the exact choice of $\mathrm{OH}$ lines is thus unimportant. The adopted $g f$-values for the $\mathrm{OH} \mathrm{A}-\mathrm{X}$ lines are taken from Gillis et al. (2001), which are based on the most recent theoretical calculations and laboratory measurements. For completeness, it is noted that a dissociation energy of $4.392 \mathrm{eV}$ (Sauval \& Tatum 1984) has been employed but it has no influence on the conclusions obtained here. The Be II 
Table 1. The predicted line strengths in pm $(=10 \mathrm{~m} \AA)$ for the OH A-X and Be lines using 1D MARCS and Holweger-Müller solar model atmospheres calculated with different combinations of microturbulence $\left(\xi_{\text {turb }}\right)$ and additional continuous opacity over standard values $\left(\Delta \kappa_{v}^{\text {cont }} / \kappa_{v}^{\text {cont }}\right)$. The "observed" equivalent widths used for the 3D calculations are those computed using $\Delta \kappa_{v}^{\text {cont }} / \kappa_{v}^{\text {cont }}=0.60$ (i.e. $60 \%$ extra opacity) and $\xi_{\text {turb }}=1.0$, as found by Balachandran \& Bell (1998).

\begin{tabular}{|c|c|c|c|c|c|c|c|}
\hline \multirow[t]{2}{*}{$\begin{array}{l}\text { line } \\
{[\mathrm{nm}]}\end{array}$} & \multirow[t]{2}{*}{$\begin{array}{l}\chi_{\mathrm{exc}} \\
{[\mathrm{eV}]}\end{array}$} & \multirow[t]{2}{*}{$\overline{\log g f}$} & \multirow[t]{2}{*}{ 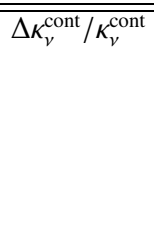 } & \multicolumn{2}{|c|}{$\begin{array}{c}W_{\lambda}(\text { MARCS }) \\
\log \epsilon_{\mathrm{O}}=8.75 \\
\log \epsilon_{\mathrm{Be}}=1.40 \\
\end{array}$} & \multicolumn{2}{|c|}{$\begin{array}{c}W_{\lambda}(\text { Holweger-Müller }) \\
\log \epsilon_{\mathrm{O}}=8.91 \\
\log \epsilon_{\mathrm{Be}}=1.40 \\
\end{array}$} \\
\hline & & & & $\begin{array}{c}\xi_{\text {turb }}=1.0 \\
{\left[\mathrm{~km} \mathrm{~s}^{-1}\right]}\end{array}$ & $\begin{array}{c}\xi_{\text {turb }}=1.2 \\
{\left[\mathrm{~km} \mathrm{~s}^{-1}\right]}\end{array}$ & $\begin{array}{c}\xi_{\text {turb }}=1.0 \\
{\left[\mathrm{~km} \mathrm{~s}^{-1}\right]}\end{array}$ & $\begin{array}{c}\xi_{\text {turb }}=1.2 \\
{\left[\mathrm{~km} \mathrm{~s}^{-1}\right]}\end{array}$ \\
\hline \multirow[t]{2}{*}{ OH 312.80} & 0.102 & -2.425 & 0.00 & 6.53 & 6.69 & 6.55 & 6.71 \\
\hline & & & 0.60 & 5.90 & 6.04 & 6.01 & 6.15 \\
\hline \multirow[t]{2}{*}{ OH 312.82} & 0.209 & -2.074 & 0.00 & 7.55 & 7.75 & 7.58 & 7.78 \\
\hline & & & 0.60 & 6.89 & 7.07 & 7.02 & 7.21 \\
\hline \multirow[t]{2}{*}{ OH 313.91} & 0.760 & -1.563 & 0.00 & 7.47 & 7.67 & 7.51 & 7.71 \\
\hline & & & 0.60 & 6.78 & 6.95 & 6.92 & 7.10 \\
\hline \multirow[t]{2}{*}{ OH 316.71} & 1.108 & -1.544 & 0.00 & 6.15 & 6.29 & 6.19 & 6.33 \\
\hline & & & 0.60 & 5.46 & 5.58 & 5.59 & 5.72 \\
\hline \multirow[t]{2}{*}{ Be II 313.10} & 0.000 & -0.468 & 0.00 & 8.79 & 8.91 & 8.63 & 8.74 \\
\hline & & & 0.60 & 7.63 & 7.73 & 7.63 & 7.73 \\
\hline
\end{tabular}

$313.10 \mathrm{~nm} g f$-value comes from the VALD database (Piskunov et al. 1995). The relevant input data is listed in Table 1.

The study presented here is done strictly differentially between the 1D and 3D cases, thereby avoiding a direct comparison with observations. Thus, in effect an intrinsic assumption for these calculations is that the investigation of Balachandran \& Bell (1998) is done correctly in terms of the comparison with observations within the framework of 1D model atmospheres and line formation. The main reason to here avoid confrontation with observations is that the current version of the $3 \mathrm{D}$ line formation code can only treat lines of one element or molecule at a time, while the $\mathrm{OH} \mathrm{A-X}$ and Be lines are located in a very crowded spectral region with contributions from many different species. An advantage with the procedure adopted here is that the impact of the 3D model atmospheres and the new low solar $\mathrm{O}$ abundance can be isolated without the possible confusion introduced by for example continuum placement and choice of transition probabilities. As there are all reasons to believe that the careful analysis of Balachandran \& Bell (1998) was done correctly, we are still in a position to evaluate the solar photospheric Be abundance and the amount of missing UV-opacity.

As the predicted line shapes are intrinsically different for the $1 D$ and $3 D$ cases even for the same line strengths, the here adopted procedure is simplified to only use equivalent widths. With the absence of direct observational confrontation, the observed line strengths of the relevant $\mathrm{OH}$ A-X lines have been estimated by redoing the analysis of Balachandran \& Bell (1998) with the continuous opacities multiplied by a factor of 1.6. The adopted solar $\mathrm{O}$ abundances for the calculations using the 1D MARCS (Asplund et al. 1997) and the Holweger-Müller (1974) solar model atmospheres are $\log \epsilon_{\mathrm{O}}=8.75$ and $\log \epsilon_{\mathrm{O}}=$ 8.91, respectively, as found by Balachandran \& Bell (1998) from the $\mathrm{OH}$ IR vibration-rotation lines with the two model atmospheres. In addition, the same calculations have been performed without the additional continuous opacity as well as for the two values of the microturbulence. The predicted 1D equivalent widths of the $\mathrm{OH}$ A-X lines for the different cases are listed in Table 1 together with the corresponding results for the Be II $313.10 \mathrm{~nm}$ line. The equivalent widths computed with a multiplication factor of 1.6 to the continuous opacity will thus function here as substitutes for the observed line strengths.

From a closer inspection of Table 1, it is clear that the two 1D model atmospheres do not yield exactly the same "observed" equivalent widths, even if Balachandran \& Bell (1998) claimed that their analysis yielded the same multiplication factor to the continuous opacities in the two cases. The reason for this relatively small discrepancy has not been identified but can probably be traced to a combination of slightly different adopted input data (continuous opacities, etc.), model atmospheres (exact version of MARCS models, pressure-integrated Holweger-Müller model atmosphere or not), overall solar chemical composition, and numerical implementation of the 1D spectrum synthesis. Assuming for the moment that the calculations with the Holweger-Müller model atmospheres are correct with $60 \%$ extra opacity, the same result would be obtained for the MARCS case with about $50 \%$ additional opacity instead. It should be emphasized that this difference has no significant impact on the conclusions regarding missing UVopacity and the photospheric Be abundance.

\section{Results}

Tables 1 and 2 list the predicted line strengths for the $\mathrm{OH}$ and Be II lines using the $1 \mathrm{D}$ and $3 \mathrm{D}$ model atmospheres, respectively. As described above, the theoretical equivalent widths calculated with 1D model atmospheres, a microturbulence of $\xi_{\text {turb }}=1.0 \mathrm{~km} \mathrm{~s}^{-1}$ and a multiplication factor of 1.6 to the continuous opacities around $313 \mathrm{~nm}$ will here function as the "observed" values to be reproduced with the predictions from the $3 \mathrm{D}$ spectrum synthesis. For the $\mathrm{OH}$ lines in $3 \mathrm{D}$ a solar $\mathrm{O}$ abundance of $\log \epsilon_{\mathrm{O}}=8.66$ has been adopted, as indicated 


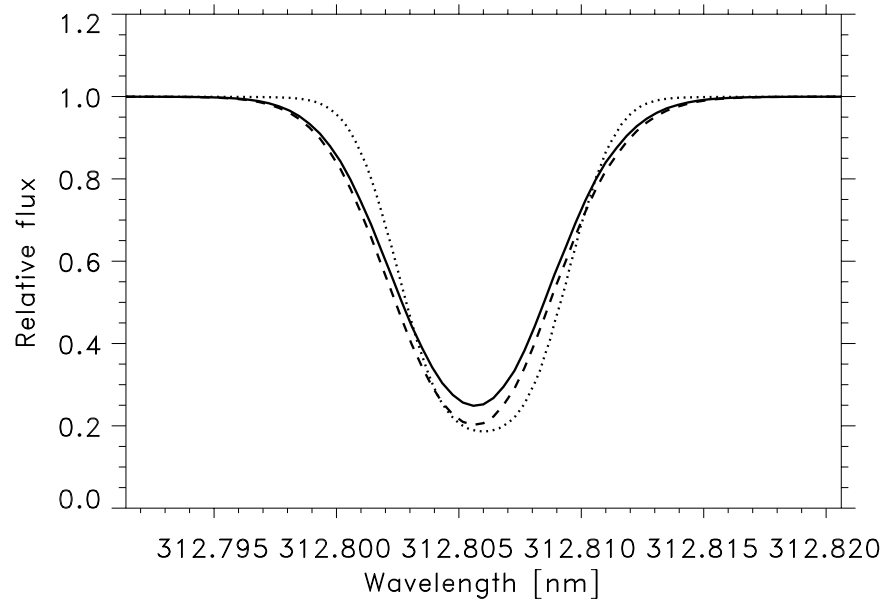

Fig. 1. The dotted line denotes the $\mathrm{OH} 312.80 \mathrm{~nm}$ line calculated with the MARCS model atmosphere using a solar oxygen abundance of $\log \epsilon_{\mathrm{O}}=8.75$, a microturbulence of $\xi_{\text {turb }}=1.0 \mathrm{~km} \mathrm{~s}^{-1}$ and $60 \%$ additional continuous opacity over the standard value computed by the spectrum synthesis program. According to Balachandran \& Bell (1998) this fits the observations very well, and is here used as a substitute to the observed profile in this crowded spectral region. The dashed line corresponds to a 3D line calculation with $\log \epsilon_{\mathrm{O}}=8.66$ and without any extra opacity. The solid line represents the 3D results with the same abundance but with $50 \%$ extra continuous opacity, which yields the same overall line strength as the here shown 1D profile. No microand macroturbulence enter the 3D calculations but the Doppler shifts caused by the convective motions make the line broader than the $1 \mathrm{D}$ case which is here shown without macroturbulence.

from our recent analysis of the $\mathrm{OH}$ vibration-rotation, the $\mathrm{OH}$ pure rotation, the forbidden [O I] lines at 630.0 and $636.3 \mathrm{~nm}$, and high-excitation, permitted O I lines (Asplund et al. 2004). It is then straightforward to compare the predicted equivalent widths using the 3D hydrodynamical model atmosphere with different multiplication factors to the continuous opacity with the "observed" line strengths of the $\mathrm{OH} \mathrm{A-X} \mathrm{lines.} \mathrm{It} \mathrm{is} \mathrm{imme-}$ diately obvious that without additional continuous opacity over those included in the spectrum synthesis package the $\mathrm{OH}$ lines are significantly too strong (Fig. 1). Using an average of the "observed" values from the Holweger-Müller and MARCS 1D model atmospheres, this missing opacity can be estimated to be about $50 \%$, which is indeed very similar to the value of $60 \%$ obtained by Balachandran \& Bell (1998).

The "observed" equivalent width of the Be II $313.0 \mathrm{~nm}$ line is independent of whether the Holweger-Müller or the MARCS 1D model atmosphere have been used to estimate it, as clear from Table $1(7.63 \mathrm{pm}=76.3 \mathrm{~m} \AA)$. In 3D, without any additional continuous opacity this line strength would imply a solar Be abundance of $\log \epsilon_{\mathrm{Be}}=1.23$. However, as clear from the comparison of the $\mathrm{OH} \mathrm{A}-\mathrm{X}$ lines, there are strong indications that the missing UV-opacity amounts to about 50\%. Taking this extra opacity into account the 3D spectrum synthesis leads to accordingly weaker lines, yielding a higher solar Be abundance of $\log \epsilon_{\mathrm{Be}}=1.38$ (Fig. 2). Combined with an estimate of the errors in the missing opacity and the overall agreement between predicted and observed line profiles (Balachandran \& Bell 1998), the final result is thus $\log \epsilon_{\mathrm{Be}}=1.38 \pm 0.09$. This

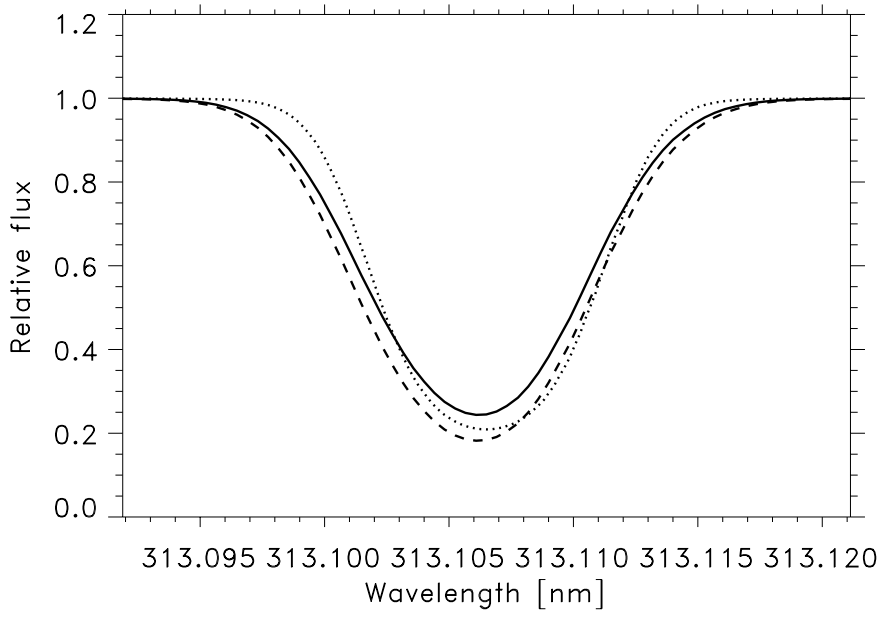

Fig. 2. The dotted line denotes the Be I $313.10 \mathrm{~nm}$ line calculated with the MARCS model atmosphere using a solar beryllium abundance of $\log \epsilon_{\mathrm{Be}}=1.40$, a microturbulence of $\xi_{\text {turb }}=1.0 \mathrm{~km} \mathrm{~s}^{-1}$ and $60 \%$ additional continuous opacity, which is required to fit the observations according to Balachandran \& Bell (1998). The dashed line corresponds to a $3 \mathrm{D}$ line calculation with $\log \epsilon_{\mathrm{Be}}=1.38$ and without any extra opacity. The solid line represents the 3D results with the same abundance but with $50 \%$ extra continuous opacity, which yields the same line strength as the here shown 1D profile.

Table 2. The predicted line strengths in pm for the $\mathrm{OH} \mathrm{A-X}$ and $\mathrm{Be}$ lines using a 3D hydrodynamical solar model atmosphere. The $\mathrm{OH}$ lines are computed for $\log \epsilon_{\mathrm{O}}=8.66$ and the $\mathrm{Be} \operatorname{line}$ for $\log \epsilon_{\mathrm{Be}}=1.38$.

\begin{tabular}{lcccc}
\hline $\begin{array}{l}\text { line } \\
{[\mathrm{nm}]}\end{array}$ & $\begin{array}{c}\chi_{\text {exc }} \\
{[\mathrm{eV}]}\end{array}$ & $\log g f$ & $\begin{array}{c}W_{\lambda} \\
\begin{array}{c}\text { cont } / \kappa_{v}^{\text {cont }} \\
=0.00\end{array}\end{array}$ & $\begin{array}{c}W_{\lambda} \\
\Delta \kappa_{v}^{\text {cont }} / \kappa_{v}^{\text {cont }} \\
=0.50\end{array}$ \\
\hline OH 312.80 & 0.102 & -2.425 & 6.38 & 5.90 \\
OH 312.82 & 0.209 & -2.074 & 7.42 & 6.91 \\
OH 313.91 & 0.760 & -1.563 & 7.29 & 6.75 \\
OH 316.71 & 1.108 & -1.544 & 5.87 & 5.34 \\
Be II 313.10 & 0.000 & -0.468 & 8.62 & 7.65 \\
\hline
\end{tabular}

best estimate using a 3D model atmosphere is indistinguishable from the meteoritic value of $\log \epsilon_{\mathrm{Be}}=1.41 \pm 0.08$ (Lodders 2003) given the uncertainties in both the photospheric and meteoritic analyses. This is even more true in the light of the proposed downward revision by 0.04 dex to the meteoritic abundance scale caused by the re-analysis of the photospheric Si abundance on which the absolute meteoritic abundance scale is anchored (Asplund 2000), which would bring the meteoritic value down to $\log \epsilon_{\mathrm{Be}}=1.37 \pm 0.08$.

The findings of Balachandran \& Bell (1998) of significant missing UV-opacity and no Be depletion within the solar convection zone throughout the 4.6 Gyr of solar evolution until today is therefore confirmed by this analysis based on a realistic 3D model atmosphere rather than 1D models.

\section{Remaining uncertainties}

In this section, uncertainties which may have a bearing on the findings are discussed. 
Model atmospheres: Available evidence from various confrontations between the predictions from the here employed 3D solar simulation with observed diagnostics all suggest that indeed the new 3D hydrodynamical model atmospheres are highly realistic, representing a significant improvement over existing 1D models. It is reassuring that the main conclusions regarding the missing UV-opacity and the solar Be abundance are robust against choice of model atmosphere, whether in 1D or in 3D.

Line broadening: It should be noted that the line strengths of the $\mathrm{OH}$ A-X lines are somewhat sensitive to the adopted microturbulence in 1D. Had instead Balachandran \& Bell (1998) employed a larger microturbulence, they would have obtained slightly smaller multiplication factors to the continuous opacity, as clear from Table 1. Due to the differential nature of this study relative to that of Balachandran \& Bell (1998), the multiplication factor estimated here using a 3D solar model atmosphere would then have been adjusted slightly accordingly. For reasonable values of the microturbulence, however, the changes are small to the estimated amount of missing UV-opacity and the derived solar Be abundance. Available solar analyses all indicate that a microturbulence of $1.0 \pm 0.1 \mathrm{~km} \mathrm{~s}^{-1}$ for flux spectra is appropriate when relying on $1 \mathrm{D}$ model atmospheres. The uncertainties attached to the exact choice of microturbulence can therefore in this case be safely ignored.

Transition probabilities: Conceivably, the adoption of erroneous transition probabilities for either the $\mathrm{OH}$ vibrationrotation or the $\mathrm{OH}$ A-X lines could be misleading, yielding incorrect estimates of the amount of missing UV-opacity, if any, and consequently the derived solar Be abundance. Fortunately, the necessary $g f$-values for both sets of lines are accurately known from theoretical calculations and laboratory measurements (Goldman et al. 1998; Gillis et al. 2001), which are very similar to those used by Balachandran \& Bell (1998) in their study. The uncertainties in the transition probabilities are sufficiently small not to compromise the conclusions presented here. Similarly, as long as consistent partition functions and molecular equilibrium constants are employed, the results are independent of such input data.

Continuum placement: A potentially more serious shortcoming of the study of Balachandran \& Bell (1998) and thus also the present analysis, is the exact placement of the continuum level. If the observed continuum is estimated to be too low, a too low abundance is derived, in particular given the fact the relevant lines for these studies are at least partly saturated. The region around the $\mathrm{OH} \mathrm{A-X}$ and Be lines at $313 \mathrm{~nm}$ is notoriously crowded and it is difficult to identify wavelength windows which traces the continuum. The problem is compounded by the fact that many of the lines in this region remain unidentified, rendering even the use of spectrum synthesis to locate small continuum portions uncertain. As this study does not involve direct comparison with observations, it relies entirely on that this part in the original Balachandran \& Bell (1998) work was carried out properly. From the relatively small wavelength regions shown in their figures it appears however that the continuum placement is essentially correct, which should minimize the uncertainties for the final results.
Non-LTE line formation for $\mathrm{OH}$ lines: While LTE is in all likelihood an excellent approximation for the formation of the $\mathrm{OH}$ vibration-rotation lines, this is not guaranteed for the $\mathrm{OH}$ A-X lines (Hinkle \& Lambert 1975). To date, very little work on non-LTE line formation has been invested for molecules with the notable exception of CO lines (e.g. Uitenbroek 2000). For OH A-X lines, the author is only aware of the very preliminary study of Asplund \& García Pérez (2001) based on only a two-level $\mathrm{OH}$ model. A fundamental obstacle in this respect is the lack of data for excitation and ionization by collisions with electrons and hydrogen atoms, even more so than for the corresponding case of atoms. With their very simplified approach Asplund \& García Pérez (2001) indeed found the possibility of significant departures from LTE for the $\mathrm{OH}$ A-X lines, amounting to about $0.2 \mathrm{dex}$ in terms of oxygen abundance for the solar case in 1D. If this significant weakening of the predicted $\mathrm{OH} \mathrm{A}-\mathrm{X}$ lines in non-LTE is correct, the conclusions regarding missing UV-opacity must be revised. Indeed, a non-LTE effect of 0.2 dex more than compensates for the effect of extra opacity. For example with an $\mathrm{O}$ abundance of $\log \epsilon_{\mathrm{O}}=8.55$, the predicted equivalent width of the $\mathrm{OH} 312.80 \mathrm{~nm}$ line with the 1D MARCs model atmosphere, $\xi_{\text {turb }}=1.0 \mathrm{~km} \mathrm{~s}^{-1}$ and no extra opacity is $5.60 \mathrm{pm}$, i.e. weaker than the "observed" line strength. In other words, this would at face value imply that there is no missing UV-opacity but rather that the known opacity sources predict too much opacity in this particular wavelength region. However, the reader is urged to be cautious and not over-interpreting the very preliminary non-LTE results of Asplund \& García Pérez (2001). It is likely that the inclusion of more levels in the $\mathrm{OH}$ model would more efficiently thermalize the molecule, bringing the results closer to LTE. It is clear that a more careful non-LTE investigation of the $\mathrm{OH}$ line formation is necessary to resolve this issue.

Non-equilibrium chemistry for $\mathrm{OH}$ molecule formation: Another crucial assumption for these types of studies is the use of instantaneous equilibrium chemistry for the molecule formation. In reality the time-scale for molecule formation is finite and if this approaches the typical convective timescale for up- and down-flows in the solar atmosphere the number density of molecules will in general be lower than expected from equilibrium chemistry. This has been shown by Asensio Ramos et al. (2003) for the case of the CO molecule in hydrodynamical models of the solar chromosphere. As for non-LTE line formation for molecules, little work has been done in this potentially crucial area. The available evidence, however, indicates that equilibrium chemistry is valid for $\mathrm{OH}$, at least in the atmospheric layers corresponding to the formation heights of the $\mathrm{OH}$ vibration-rotation and $\mathrm{A}-\mathrm{X}$ lines in the Sun (Sánchez Almeida et al. 2001; Asensio Ramos et al. 2003; Asensio Ramos \& Trujillo Bueno 2003). In addition, these effects only influence the present study in as far as the two types of lines are formed in different layers with a differing degree of appropriateness for the assumption of equilibrium chemistry: if both the vibration-rotation and A-X lines have the same typical formation heights any non-equilibrium molecule formation will affect both similarly, leaving the estimate of the missing UV-opacity and the solar Be abundance intact. 
Non-LTE line formation for Be lines: Finally, possible departures from LTE for the line formation of the Be II lines can of course influence the derived solar Be abundance. Fortunately, the two main non-LTE effects for these lines over-ionization and over-excitation - essentially compensate each other for the solar case, coincidentally leaving the nonLTE Be abundance very similar to the LTE value: $\Delta\left(\log \epsilon_{\mathrm{Be}}\right)<$ 0.02 dex (García Pérez et al., in preparation). Thus, the finding of no significant Be depletion is safe to departures from LTE in the Be line formation.

\section{Conclusions}

In the present article a re-analysis of the work by Balachandran \& Bell (1998) has been performed employing a state-of-theart 3D hydrodynamical solar model atmosphere (Asplund et al. 2000a) instead of traditional 1D hydrostatic models. Several of the uncertainties attached to the analysis by Balachandran \& Bell (1998) are thus removed and the important results placed on a more firm footing. In addition, several of the outstanding sources of uncertainties have here been discussed at some length and, with one notable exception, found to be in most likelihood unimportant for the basic findings. The conclusions by Balachandran \& Bell (1998) of a significant missing UVopacity around $313 \mathrm{~nm}$ and a photospheric Be abundance in very close agreement with the meteoritic value measured in CI-chondrites are therefore confirmed by this study based on a more realistic model atmosphere. According to this study, the additional opacity amounts to about $50 \%$ in this particular wavelength region, which is only slightly smaller than Balachandran \& Bell (1998) estimated using 1D model atmospheres (about 60\%).

Although this study does not shed further light on the nature of the opacity source causing the missing UV-opacity it is likely that it can be traced to photo-ionization of Fe I. Using data from the Iron Project, Bell et al. (2001) indeed found that such unaccounted for Fe I bound-free cross-sections are close to explaining the missing $60 \%$ continuous opacity estimated by Balachandran \& Bell (1998). This will ring even more true with the slightly smaller multiplication factors derived here.

The situation related to the depletion of the light elements in the solar photosphere can thus be summarized as follows: while lithium has been destroyed by a factor of about 140 (Lodders 2003; Asplund et al. 2003a), neither the photospheric beryllium nor the boron abundances appear to have been significantly modified throughout the solar evolution to the present day. As described in the Introduction, no standard model of stellar evolution can account for this depletion pattern. Furthermore, is poses a severe challenge for non-standard models such as slow rotational-induced mixing below the convection zone. Indeed, it is a benchmark which any such models must successfully be tested against before they can be trusted for other types of stars.

Acknowledgements. This work has been supported by the Swedish Research Council (F990/1999) and the Australian Research Council (DP0342613). The author gratefully acknowledges financial support from the local organizing committee of the conference $C N O$ in the Universe where these results were presented.

\section{References}

Allende Prieto, C., Lambert, D. L., \& Asplund, M. 2001, ApJ, 556, L63

Allende Prieto, C., Asplund, M., García López, R. J., \& Lambert, D. L. 2002a, ApJ, 567, 544

Allende Prieto, C., Lambert, D. L., \& Asplund, M. 2002b, ApJ, 573, L137

Asensio Ramos, A., \& Trujillo Bueno, J., in Solar polarization, ed. J. Trujillo Bueno, \& J. Sánchez Almeida, ASP Conf. Ser. 307, 195

Asensio Ramos, A., Trujillo Bueno, J., Carlsson, M., \& Cernicharo, J. 2003 ApJ, 588, L61

Asplund, M. 2000, A\&A, 359, 755

Asplund, M., \& García Pérez, A. E. 2001, A\&A, 372, 601

Asplund, M., Gustafsson, B., Kiselman, D., \& Eriksson, K. 1997, A\&A, 318, 521

Asplund, M., Nordlund, Å., Trampedach, R., \& Stein, R. F. 1999, A\&A, 346, L17

Asplund, M., Nordlund, Å., Trampedach, R., Allende Prieto, C., \& Stein, R. F. 2000a, A\&A, 359, 729

Asplund, M., Nordlund, ̊̊., Trampedach, R., \& Stein R. F. 2000b, A\&A, 359, 743

Asplund, M., Carlsson, M., \& Botnen, A. V. 2003a, A\&A, 399, L31

Asplund, M., Grevesse, N., Sauval, J., \& Allende Prieto, C. 2003b, A\&A, submitted

Asplund, M., Grevesse, N., Sauval, J., \& Allende Prieto, C. 2003c, A\&A, submitted

Asplund, M., Grevesse, N., Sauval, J., Allende Prieto, C., \& Kiselman, D. 2004, A\&A, 417, 751

Balachandran, S., \& Bell, R. A. 1998, Nature, 392, 23

Bell, R. A., Balachandran, S., \& Bautista, M. A. 2001, ApJ, 546, L46

Chiemlewski, Y., Müller, E. A., \& Brault, J. M. 1975, A\&A, 42, 37

Cunha, K., \& Smith, V. V. 1999, ApJ, 512, 1006

Deliyannis, C. P., Pinsonneault, M. H., \& Charbonnel, C. 2000, in The light elements and their evolution, ed. L. da Silva, M. Spite, \& J. R. de Medeiros, IAU Symp. 198, 61

Gillis, J. R., Goldman, A., Stark, G., \& Rinsland, C. P. 2001, JQRST, 68,225

Goldman, A., Schoenfeld, W. G., Goorvitch, D., et al. 1998, JQRST, 59,453

Gustafsson, B., Bell, R. A., Eriksson, K., \& Nordlund, Å. 1975, A\&A, 42, 407

Gustafsson, B., \& Bell, R. A. 1979, A\&A, 74, 313

Hinkle, K. H., \& Lambert, D. L. 1975, MNRAS, 170, 447

Holweger H., \& Müller E. A. 1974, Sol. Phys., 39, 19

Hummer, D. G., Berrington, K. A., Eissner, W., et al. 1993, A\&A, 279, 298

Kiselman, D. 1997, ApJ, 489, L107

Kiselman, D., \& Carlsson, M. 1996, A\&A, 311, 680

Kohl, J. L., Parkinson, W. H., Withbrow, G. L. 1977, ApJ, 212, L101

Lodders, K. 2003, ApJ, 591, 1220

Mihalas, D., Däppen, W., Hummer, D. G. 1988, ApJ, 331, 815

Müller, E. A., Peytremann, E., \& de la Reza, R. 1975, Sol. Phys., 41, 53

Piskunov, N. E., Kupka, F., Ryabchikova, T. A., Weiss, W. W., \& Jefferey, C. S. 1995, A\&AS, 112, 525

Sauval, A. J., \& Tatum, J. B. 1984, ApJS, 56, 193

Sánchez Almeida, J., Asensio Ramos, A., Trujillo Bueno, J., \& Chernicharo, J. 2001, ApJ, 555, 978

Shchukina, N., \& Trujillo Bueno, J. 2001, ApJ, 550, 970

Stein, R. F., \& Nordlund, Å. 1998, ApJ, 499, 914

Uitenbroek, H. 2000, ApJ, 536, 481

Vernazza, J. E., Avrett, E. H., \& Loeser, R. 1976, ApJS, 30, 1

Zhai, M., \& Shaw, D. 1994, Meteoritics, 29, 607 